\section{Chylous Ascites and Cervical Lymphadenopathy: Variable Presentations of Perinatal Tuberculosis in Extreme Preterm Twins}

Abdominal tuberculosis has been widely reported as a cause for chylous ascites in adults and children [1]; though, not in neonates and young infants. We report an extreme preterm infant, who presented at 2.5 month of age with chylous ascites caused by perinatal tuberculosis.

A 2.5-month-old girl presented with fever, excessive crying and grunting. The infant was first of twins born at 27 weeks with birthweight of $870 \mathrm{~g}$. Both twins had received surfactant therapy, and ventilation of 48 hours followed by continuous positive airway pressure (CPAP) for five weeks. The second twin girl had birthweight of $1090 \mathrm{~g}$. She had enterobacter sepsis in the third week of nursery stay from which she recovered after receiving intravenous antibiotics. She had also received packed cell transfusion for anemia. The first twin did not require any transfusion. Both infants were fed initially with pasteurized donor milk (PDHM) followed by mother's own milk. They gained weight along the 10th centile for preterm Fenton growth chart. At the time of discharge, the first and second twin weighed $1.58 \mathrm{~kg}$ and $1.6 \mathrm{~kg}$, respectively. Both twins received iron, calcium and vitamin D supplements at discharge at corrected gestational age of 35 weeks. Their mother received antibiotics for fever and cesarian section wound dehiscence in the second postpartum week.

At the time of re-admission, the infant was febrile and her systemic examination was normal. The investigations showed leukocytosis $\left(28.9 \times 10^{9} / \mathrm{L}\right)$ with raised C-reactive protein (CRP, $97.5 \mathrm{mg} / \mathrm{L}$ ). The infant was started on intravenous fluids and antibiotics. The fever spikes reduced and CRP showed declining trend over the next week. Blood and urine cultures were reported sterile. In the second week of admission, the infant started developing abdominal distention. Her abdominal ultrasound showed moderate ascites with multiple necrotic abdominal lymph nodes. An abdominal paracentesis showed milky ascitic fluid. Ascitic fluid microscopy showed high leukocytes count $\left(3.6 \times 10^{9} / \mathrm{L}\right)$ with lymphocyte predominance $(95 \%)$. Triglycerides in ascitic fluid were high $(836 \mathrm{mg} / \mathrm{dL})$, suggestive of chylous ascites. The infant was started on octreotide infusion and medium chain triglyceride (MCT)- based formula feeds. The ascitic fluid was negative for acid fast bacilli, bacterial culture and polymerase chain reaction (PCR) for cytomegalovirus and Epstein Barr virus. GeneXpert for tuberculosis was reported as positive. A tuberculin skin test was negative after 72 hours. A computerized tomography (CT) scan of abdomen and chest revealed multiple necrotic lymph nodes in gastro-hepatic, celiac, periportal and retroperitoneal areas with necrotic lesions in spleen, and moderate ascites. A repeat ascitic fluid and gastric lavage sample for GeneXpert was also reported positive.

The infant was started on anti-tuberculosis therapy (isoniazid, rifampicin, pyrazinamide and ethambutol). Repeat abdominal paracentesis days later showed reduced triglycerides content $(230 \mathrm{mg} / \mathrm{dL})$. Octreotide infusion was stopped after seven days. Over the next four weeks, the abdominal circumference returned back to normal on MCT-based formula feeds. Family screening for tuberculosis was advised but got delayed due to coronavirus disease (COVID-19) related lockdown, and non-availability of non-emergency laboratory services.

At five months of age, the second twin was noted to have cervical lymphadenopathy. An excisional biopsy of lymph node showed caseating granuloma with Mycobacterium tuberculosis grown on Bactec MGIT system. The mother's sputum tested positive for tuberculosis on GeneXpert. The second twin and the mother were also started on four drug anti-tuberculosis treatment. The twin girl with chylous ascites was gradually shifted from MCT-based formula to term formula after 12 weeks. The infant had no ascites and was gaining weight at four months of follow up.

In addition to the known etiological factors such as congenital lymphatic malformations or surgical injury to the lymphatic system and infections, in a large number of cases the cause for chylous ascites remains unidentified [2]. The presence of necrotic lymph nodes in the ultrasound (and later in the CT scan) prompted us to investigate for tuberculosis as the cause of chylous ascites. We used GeneXpert for the diagnosis of tuberculosis in this child as gene-based tests have been shown to increase the diagnostic yield in infantile and childhood tuberculosis [3].

In spite of widely reported criteria for differentiation between congenital and postnatal onset of tuberculosis and its subsequent modification, tuberculosis infection in utero can be indistinguishable from perinatal or early post-partum infection $[4,5]$. Therefore, we have used the term 'perinatal tuberculosis' for both the twins. As such, the differentiation is only of epidemiological importance and their mode of presentation, treatment, and immediate prognosis do not differ [6]. In our case, the mother had fever during post-partum period, the cause for which was attributed to the wound dehiscence. However, it is likely that the mother could have contracted tuberculosis infection during this period. Women in early postpartum period are twice as likely to develop tuberculosis as nonpregnant women. In our case, both twins did not have any evidence of tuberculosis at birth as well as during their prolonged nursery stay. The identification of tuberculosis bacilli in mother itself indicates her being the source of TB leading to affection of both infants.

We wish to sensitize the readers about the need to also consider tuberculosis as a cause of chylous ascites in infants, 
especially in high disease burden countries.

Suresh Birajdar, ${ }^{1 *}$ Sachin Murade, ${ }^{2}$ Sharayu Kothavade, ${ }^{2}$ Leena Gajbhar, ${ }^{3}$

RAVINDRA PATILGHuge ${ }^{4}$

From ${ }^{1}$ Department of Neonatology and ${ }^{3}$ Microbiology, Motherhood Hospital, Kharghar, Navi Mumbai;

${ }^{2}$ Department of Pediatrics, Chirayu Children's Hospital,

Panvel; and

${ }^{4}$ Lotus Imaging Clinics, Kharghar, Navi Mumbai;

Maharashtra.

\section{REFERENCES}

*sbirajdar@gmail.com

1. Mallick B, Mandavdhare HS, Aggarwal S, et al. Mycobacterial chylous ascites: Report of three cases and systematic review.
Ther Adv Infect Dis. 2018;5:69-75.

2. Lopez-Gutierrez JC, Tovar JA. Chylothorax and chylous ascites: Management and pitfalls. Semin Pediatr Surg. 2014;23:298-302.

3. Atherton RR, Cresswell FV, Ellis J, et al. Xpert MTB/RIF Ultra for tuberculosis testing in children: A Mini-Review and Commentary. Front Pediatr. 2019;7:34.

4. Dewan P, Gomber S, Das S. Congenital tuberculosis: A rare manifestation of a common disease. Paediatr Int Child Health. 2014;34:60-2.

5. Pal P, Ghosh A. Congenital tuberculosis: late manifestation of the maternal infection. Indian J Pediatr. 2008;75:516-8.

6. Hageman JR. Congenital and perinatal tuberculosis: Discussion of difficult issues in diagnosis and management. $\mathrm{J}$ Perinatol. 1998;18:389-94.

\section{An Unusual Case of Auto-Immune Hemolytic Anemia}

Autoimmune hemolytic anemia in association with insect bites is a rare presentation, but delay in diagnosis can cause significant morbidity and mortality. Here, we report a case of Coombs positive hemolytic anemia after a wasp bite.

A 12-year-old male without significant past medical history was transferred to our hospital by his primary care physician with persistent fatigue, bilateral lower extremity pain, and history of undocumented fever. His laboratory work-up was remarkable for hemolytic anemia with hemoglobin of $4.5 \mathrm{~g} / \mathrm{dL}$, reticulocyte count of $6.4 \%$, elevated indirect bilirubin of $9.2 \mathrm{mg} / \mathrm{dL}$, and lactate dehydrogenase (LDH) of $1702 \mathrm{U} / \mathrm{L}$. His serum creatinine kinase was also elevated at $1927 \mathrm{U} / \mathrm{L}$.

Upon arrival to our hospital, he was febrile $\left(102.9^{\circ} \mathrm{F}\right)$ and had tachycardia. On physical exam, he was icteric and noted to have $1 \times 2 \mathrm{~cm}$ and $1 \times 3 \mathrm{~cm}$ eschars with surrounding induration on left side of his abdomen. Direct anti-globulin test (DAT) was positive with anti-IgG reagent $(3+)$. He was admitted to the pediatric intensive care unit (PICU) with a cinical diagnosis of auto-immune hemolytic anemia (AIHA) due to insect bite. Mother gave a history of seeing wasps in the house on the day of bite and per the toxicologists, the rash was consistent with a hymenoptera bite.

Upon admission, he was given blood transfusion and started on methylprednisolone. He was also started on empiric vancomycin and cephalosporin, which were discontinued 48 hours later after negative blood cultures. During his course in the ICU, he continued to require blood transfusion with ongoing drop in hemoglobin. After three days of steroids, hemolysis stopped and his hemoglobin stabilized at $8.9 \mathrm{~g} / \mathrm{dL}$. Creatinine kinase, LDH, and reticulocyte count also decreased. He was discharged home after two days on a steroid taper with recommendations for outpatient follow up. Infection was ruled out on the basis of negative cultures. No other known exposure to a new medicine was elicited. The rash being localized, specific history to a topical agent was asked but was negative. Moreover, DAT positive for IgG reagent in the presence of history of exposure to wasp in the house was suggestive of warm autoimmune hemolytic anemia (AIHA).

AIHA is defined as the destruction of circulating red blood cells (RBCs) in the setting of anti-RBC autoantibodies that optimally react at $37^{\circ} \mathrm{C}$ [1]. About $50 \%$ of the warm AIHA cases are called primary because no specific etiology can be found, whereas the rest are recognized as secondary to lymphoproliferative syndromes, malignant diseases, rheumatologic diseases, especially systemic lupus erythematosus, infections (mostly viral), drugs, or a previous transfusion or transplantation. Laboratory work-up of the patient was not suggestive of any of these secondary causes.

This presentation caused by insect bite is a rare clinical entity. The exact mechanism of this type of hemolytic anemia is unclear. However, it has been proposed that the toxin from the insect bite alters the red blood cell membrane structure making it more vulnerable to complement-mediated lysis [1]. Medical management primarily consists of supportive treatment. General consensus for first line pharmacologic treatment is glucocorticoids. It is believed that steroids not only decrease antibody production, but also suppress the effect of tissue macrophage phagocytosis and direct effect on auto-antibody red blood cell affinity $[2,3]$.

Such presentations of AIHA due to insect bites can pose a diagnostic challenge and can potentially be fatal. This case demonstrates the importance of a high level of suspicion to allow for timely recognition and intervention $[2,4]$.

$$
\begin{array}{r}
\text { LAURA LeE BENEKe, }{ }^{1} \text { PADMA GARG }{ }^{2 *} \\
{ }^{1} \text { School of Medicine; } \text { and } \\
{ }^{2} \text { Department of Pediatric Critical Care, }
\end{array}
$$

University of Mississippi Medical Center; Jackson, MS, USA. *drpadmagarg@gmail.com 